\title{
DESENVOLVIMENTO, AUTOMAÇÃO E DESEMPENHO DE UM CONSOLIDÔMETRO COM INTERFACE HOMEM-MÁQUINA
}

\author{
Reginaldo Barboza da Silva ${ }^{(1)^{*}}$, Batista Junior Masqueto ${ }^{(2)}$, Kléber Pereira Lanças ${ }^{(3)}$ e \\ Francisca Alcivania de Melo Silva ${ }^{(1)}$
}

(1) Universidade Estadual Paulista, Campus Experimental de Registro, Registro, São Paulo, Brasil.

(2) Masquetto Automação \& Equipamentos, Botucatu, São Paulo, Brasil.

(3) Universidade Estadual Paulista, Faculdade de Ciências Agronômicas, Campus de Botucatu, Botucatu, São Paulo, Brasil.

* Autor correspondente.

E-mail: rbsilva@registro.unesp.br

\begin{abstract}
RESUMO
O ensaio de consolidação preconiza a utilização de um consolidômetro. Esse equipamento, até então, não era produzido e comercializado no Brasil. Os modelos não automatizados disponíveis para importação, apesar da proposta de baixo custo, ainda são rústicos e necessitam de contínua calibração dos níveis de pressão durante a realização do ensaio. A exclusividade e intervenção de um técnico durante todo o ensaio, associada à precária coleta de dados nesses modelos, ainda são os principais fatores que têm inviabilizado a consolidação desse ensaio na ciência do solo brasileira. Como alternativa a esses problemas, este trabalho teve por objetivos desenvolver e automatizar um consolidômetro a partir de um Controlador Lógico Programável (CLP) com interface homem-máquina (IHM). O equipamento é constituído de um gabinete de metal que aloja conjuntos de dispositivos pneumáticos, eletrônico-digital e atuadores de força e posição. $O$ funcionamento de cada dispositivo de forma isolado ou conjugado é gerenciado por meio de um software em linguagem de programação ladder, que, a partir de um CLP com IHM incorporada, possibilita armazenar instruções e implementar funções. A interface entre o PC e o consolidômetro é feita pelo software CA-Linker, v 1.0, projetado especificamente para o equipamento. O uso do CLP com IHM incorporada permitiu o desenvolvimento e a automação do consolidômetro. $O$ desempenho e a eficiência do conjunto de dispositivos (pneumáticos, eletrônico-digital e atuadores de força e pressão) foram comprovados pelos excelentes resultados dos valores de deformação e pressão obtidos em função do tempo e, principalmente, do comportamento da curva de compressão, gerada pelos ensaios de compressão.
\end{abstract}

Palavras-chave: compressibilidade do solo, Controlador Lógico Programável, ensaio de compressão uniaxial, pressão de pré-consolidação. 


\title{
ABSTRACT: DEVELOPMENT, AUTOMATION AND PERFORMANCE OF A CONSOLIDOMETER WITH HUMAN-MACHINE INTERFACE
}

\begin{abstract}
To run a consolidation test, it is necessary to use a consolidometer. This equipment, however, had not been produced and sold in Brazil until some years ago. Non-automatic models available for import, although of low cost, are nevertheless rustic and need continuous calibration of pressure levels during the consolidation test. The exclusiveness and intervention of a technician throughout the test, associated with the precarious acquisition of data in these models, are still major factors that have made consolidation of this test impracticable in Brazilian soil science. As an alternative to these problems, the aim of this study was to develop and to automatize a consolidometer through a Programmable Logic Controller (PLC) with human-machine interface (HMI). This piece of equipment consists of a metal cabinet that houses sets of pneumatic and electronic-digital devices, and force and position actuators. The operation of each device in an isolated or combined manner is managed by software in ladder programming language, which makes it possible to store instructions and to implement functions from a PLC with incorporated HMI. The interface between the PC and the consolidometer was developed by "CA-Linker" software, $v$ 1.0, designed specifically for the equipment. The use of a PLC with incorporated HMI allowed the development and automation of the consolidometer. The performance and efficiency of the set of devices (pneumatic and electronic-digital devices, and force and position actuator) were confirmed by the excellent results of the strain values obtained as a function of time, and especially the results of the compression curve generated from the compression tests.
\end{abstract}

Keywords: preconsolidation pressure, Programmable Logic Controller, soil compressibility, uniaxial compression test.

\section{INTRODUÇÃO}

$\mathrm{Na}$ última década, com o avanço da agricultura e das técnicas de precisão, o emprego de máquinas e implementos modernos, mais potentes e pesados, têm levado técnicos e pesquisadores da ciência do solo a desenvolver novos métodos de análise, assim como propor a inclusão de atributos mecânicos e dinâmicos em estudos de avaliação da estrutura dos solos agrícolas (Dias Júnior e Pierce, 1995; Peng et al., 2004; Araujo-Junior et al., 2008; Silva et al., 2010; Pais et al., 2011; Iori et al., 2012a; Pires et al., 2012). Iniciativas como essas têm feito da modelagem e da predição da Capacidade de Suporte de Carga do Solo (CSCS) uma proposta viável e exequível, mesmo que ainda praticada por um grupo restrito de pesquisadores no Brasil (Silva et al., 2003a; Severiano et al., 2008; Iori et al., 2013; Dias Júnior et al., 2005; Araujo-Junior et al., 2011; Pais et al., 2013).

A utilização de modelos de CSCS para predizer os efeitos do tráfego sobre a estrutura do solo preconiza a utilização da pressão de pré-consolidação $\left(\sigma_{\mathrm{p}}\right)$ (Kamimura et al., 2012; Ajayi et al., 2013; Martins et al., 2013; Severiano et al., 2013). A pressão de pré-consolidação é uma propriedade mecânica do solo obtida em laboratório e é indicadora da pressão máxima que pode ser aplicada ao solo de maneira a não compactar adicionalmente o solo (Dias Júnior e Pierce, 1996).

Em estudos aplicados, a pressão de pré-consolidação tem sido georreferenciada por meio do uso do Sistema de Posicionamento Global (GPS) e tem permitido a criação de mapas de tráfegos, que têm sido empregados para identificar, em áreas agrícolas, regiões de maior resistência mecânica do solo (Silva et al., 2009; Kamimura et al., 2013). Esses mapas associados a modelos preditivos, que ajustados em razão do teor de água (Dias Júnior, 1994; Silva et al., 2003b; Ajayi et al., 2010; Ajayi et al., 2011; Severiano et al., 2011; Iori et al., 2012b; Souza et al., 2012) tem se constituído em poderosa ferramenta nos estudos de interação máquina-solo.

Todavia, a obtenção da pressão de pré-consolidação se dá a partir de um método denominado ensaio de consolidação, cuja definição, de acordo com a publicação EM 1110-2-1906 (U.S. Army Engineer Manual, 1970), proposta pela CECW-EC do Laboratory Soils Testing, consiste no processo de transferência gradual de uma carga aplicada à estrutura do solo, até que todos os vazios do solo sejam comprimidos.

O ensaio de consolidação depende da utilização de equipamentos específicos, como um consolidômetro. Esse equipamento, até então, não era produzido comercialmente no Brasil e os modelos disponíveis no exterior, mesmos os não automatizados, apresentam valor elevado, o que dificulta a sua utilização. Esses motivos têm inviabilizado, em alguns casos, o acesso a informações da CSCS, tanto por parte dos pesquisadores como por parte dos agricultores e, de maneira geral, têm comprometido obter e divulgar essa propriedade mecânica, tão importante em estudos de avaliação e predição do processo de compactação dos solos agrícolas brasileiros.

Buscando alternativa para esse problema, Silva et al. (2007) e Figueiredo et al. (2011), a exemplo dos poucos aparelhos importados e 
em funcionamento no Brasil, desenvolveram os primeiros protótipos de funcionamento manual. Apesar de a tecnologia empregada nas duas propostas ser totalmente nacional e de baixo custo, a rusticidade, a necessidade contínua de calibração dos níveis de pressão a serem aplicadas, a exclusividade e intervenção do técnico durante todo o ensaio, associada à precária coleta de dados, que são inexistentes nos modelos (consolidômetros) não automatizados, ainda são os principais fatores que têm inviabilizado a popularização e rotina desse método nos centros e laboratórios de solo do país.

Uma alternativa a essas questões é, portanto, o desenvolvimento e a automação de um equipamento por meio da utilização de Controladores Lógicos Programáveis (CLP) e recursos tecnológicos incorporados de interface homem-máquina (IHM). O CLP é um equipamento eletrônico digital com hardware e software compatíveis com aplicações industriais. Conforme o National Electrical Manufactures Association (NEMA), é um aparelho eletrônico digital que utiliza uma memória programável para armazenar internamente instruções e implementar funções específicas, como lógica, sequenciamento, temporização, contagem e aritmética, controlando, por meio de módulos de entradas e saídas analógicas e digitais, vários tipos de máquinas ou processos.

Este trabalho teve como objetivo desenvolver um Consolidômetro Automático com Interface HomemMáquina incorporado para realizar ensaios de compressão uniaxial e possibilitar, em tempo real, o processamento, a sistematização e o gerenciamento de índices físicos e mecânicos do solo.

\section{MATERIAL E MÉTODOS}

\section{Projeto e desenvolvimento}

O projeto e equipamento, desenvolvidos em parceria com a Masquetto Automação e Equipamentos ${ }^{\circledR}$, foi motivado pela demanda das atividades do Pós-Doutorado do primeiro autor, desenvolvido na Universidade Estadual Paulista, Campus de Botucatu. O consolidômetro é constituído de um gabinete de metal que aloja os seguintes conjuntos de dispositivos: pneumáticos, eletrônico-digital e atuadores de força e posição (Figura 1).

\section{Dispositivos pneumáticos}

a. Um atuador pneumático de simples ação por membrana com pressão máxima de trabalho de 10 bar (145 psi). O diâmetro da área útil é de $110 \mathrm{~mm}$ e o curso de deslocamento máximo é de $21 \mathrm{~mm}$;

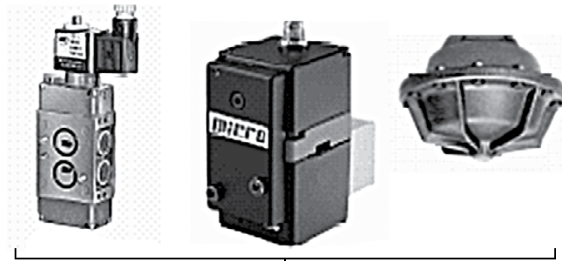

(a)

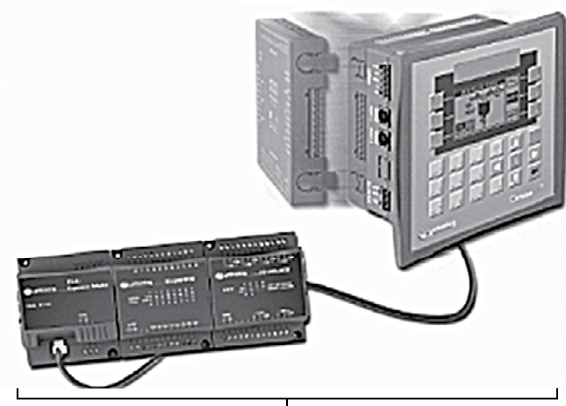

(b)

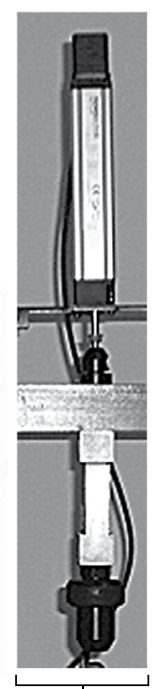

(c)
Figura 1. Conjuntos de dispositivos constituintes do equipamento. (a) dispositivos pneumáticos, (b) dispositivos eletrônico-digital e (c) dispositivos de atuação de força e deformação.

b. Uma reguladora de pressão proporcional que é controlada eletronicamente com base na tecnologia piezoelétrica. Atua instantaneamente, limitando uma vazão de ar em resposta a uma alteração de sinal de comando de voltagem de baixa potência. A máxima pressão de entrada e a de saída são de 10 bar (145 psi) e 8 bar (116 psi), respectivamente; e

c. Uma válvula direcional de atuação pneumática e elétrica. A pressão de trabalho é de 0 a 8 bar e com uma vazão nominal de $1.000 \mathrm{~L} \mathrm{~min}^{-1}$ $(1,016 \mathrm{Cv})$.

\section{Dispositivos eletrônico-digitais}

Um Controlador Lógico Programável (CLP) com Interface Homem-Máquina (IHM) incorporada. O display gráfico de dimensão de $8 \times 22 \mathrm{~mm}$ tem resolução de $128 \times 64$ pixel e apresenta um teclado numérico com funções legendadas. Dependendo da configuração, pode disponibilizar até duas entradas e duas saídas analógicas e 16 entradas e 14 saídas digitais. Contemplam ainda duas portas RS232 e a saída para Ethernet, como opções de comunicação.

\section{Dispositivos de força e deformação (atuadores)}

a. Uma célula de carga de escala nominal de $1.000 \mathrm{kgf}$, sensibilidade nominal de $2 \mathrm{mV} / \mathrm{V}$, alimentação de 10 a 12 Vcc, erro global (linearidade, histerese e repetibilidade) menor que $0,1 \%$ e construída em aço liga; e 
Quadro 1. Detalhamento da rotina incorporada ao software para determinar o tempo de consolidação e avanço dos carregamentos, com vistas ao atendimento da pressuposição de Taylor (1948)

\begin{tabular}{|c|c|c|c|c|c|}
\hline \multirow[t]{2}{*}{$\mathbf{P}$} & \multirow[t]{2}{*}{$\mathbf{T}$} & \multirow[t]{2}{*}{$\mathbf{D}$} & \multicolumn{3}{|c|}{ Procedimento a seguir } \\
\hline & & & A & B & C \\
\hline \multirow[t]{5}{*}{$\mathrm{kPa}$} & $\mathrm{s} / \mathrm{min}$ & $\mathrm{mm}$ & & & \\
\hline & $0 ”$ & 0 & Calcula-se a diferença entre as & Calculam-se $5 \%$ (IR) da & Se a diferença entre as \\
\hline & $15 ”$ & 0,5 & deformações referentes aos tempos & deformação referente ao tempo & deformações referente aos \\
\hline & $30 ”$ & & de 4' e 2', isto é: 5 - 2,8 = 2,2 mm & de 4', isto é: $0,05 \times 5=0,25 \mathrm{~mm}$ & tempos de 4' e 2' $(2,2 \mathrm{~mm})$ for \\
\hline & $30^{\circ}$ & 1 & & & maior que os $5 \%$ da deformação \\
\hline \multirow{6}{*}{$\underline{25}$} & $1^{\prime}$ & 2 & & & referente ao tempo 4' $(0,25)$, o \\
\hline & 2 ' & 2,8 & & & $\begin{array}{c}\text { ensaio continua e são refeitos } \\
\text { os cálculos para o tempo }\end{array}$ \\
\hline & $\underline{4}$ & $\underline{5}$ & \multicolumn{2}{|c|}{$2,2 \mathrm{~mm}>0,25 \mathrm{~mm}$} & seguinte, isto é, 8'. Continua o \\
\hline & $8^{\prime}$ & 10 & \multirow{2}{*}{\multicolumn{2}{|c|}{$\downarrow \downarrow \downarrow$}} & procedimento até o tempo de 15 , \\
\hline & 15 & 12 & & & $\begin{array}{c}\text { se for necessário. Caso contrário, } \\
\text { se avança para o próximo }\end{array}$ \\
\hline & & & \multicolumn{2}{|c|}{$\begin{array}{c}\text { O programa orienta o equipamento a permanecer na mesma pressão } \\
\text { e avançar para o próximo tempo, isto é, 8'. }\end{array}$} & carregamento. \\
\hline
\end{tabular}

P: Pressão aplicada na amostra; T: tempo de consolidação do corpo-de-prova; D: deformação do corpo-de-prova.

Foram atendidas as recomendações do método proposto por Bowles (1986), modificadas por Dias Júnior (1994), que preconizam a aplicação dos seguintes níveis de pressão: 25, 50, 100, 200, 400, 800 e $1.600 \mathrm{kPa}$, até que $90 \%$ da deformação máxima na amostra seja obtida (Taylor, 1948). Para atender a pressuposição de Taylor (1948), uma rotina foi programada em ladder e incorporada no CLP (Quadro 1), cujos cálculos e comandos supervisionam o tempo de consolidação dentro de cada carregamento (nível de pressão), assim como a tomada de decisão de avanço para o próximo tempo e, ou, carregamento até o término do ensaio.

Para atender as pressuposições descritas anteriormente, foi feito um comparativo entre o valor absoluto $(0,25 \mathrm{~mm})$ referente a um índice relativo (IR); nesse caso, de $5 \%$ da deformação do tempo corrente e do valor absoluto $(2,2 \mathrm{~mm})$ referente à diferença de deformação do tempo corrente e do anterior (Quadro 1). No equipamento, é sugerida uma rotina-padrão, cuja recomendação é de 5 \% (Figura 3 ), porcentagem essa definida com base em resultados de muitos ensaios já realizados e de informações obtidas por comunicação pessoal de distintos pesquisadores. Essa sugestão está fundamentada em testes realizados com distintas classes texturais, que evidenciaram que solos mais argilosos e mais úmidos, por causa da maior compressibilidade, demandaram valores de IR da ordem de 4 a $6 \%$.

Ressalta-se que rotinas programadas e embarcadas no equipamento, assim como os recursos da interface homem-máquina (IHM) do controlador (CLP), permitem que esse parâmetro de entrada seja reconfigurado pelo usuário para valores entre 1 e $12 \%$ (Figura 3), conforme tipo de solo, teor de água e experiência do pesquisador, antes da realização de cada ensaio. Durante a configuração do ensaio de consolidação, esse procedimento é feito integralmente por meio do $\mathrm{PC}$, utilizando-se de telas e ambiente do CA-Linker (Figura 3).

O equipamento, por meio do CA-Linker, possibilita ao técnico uma configuração distinta das sugeridas na literatura (Dias Júnior, 1994), permitindo independência no nível de pressão e tempo de carregamento. Para um corpo-de-prova de dimensões de 36,3 , esse recurso permite ao equipamento autonomia para elevar o corpo-de-prova a um estado de consolidação desejado, com níveis de pressão variando entre 1 e $1600 \mathrm{kPA}$. O tempo máximo possível para cada ensaio, independentemente do carregamento é de $12.600 \mathrm{~s}(3,5 \mathrm{~h})$.

\section{RESULTADOS E DISCUSSÃO}

Nas figuras 4 e 5, estão apresentados o protótipo do equipamento e os cortes esquemáticos com detalhes dos seus dispositivos e acessórios. Com dimensões reduzidas e formato compacto, o aparelho apresenta um peso de aproximadamente $15 \mathrm{kgf}$. Acompanham o equipamento os seguintes acessórios: dispositivos de acondicionamento do corpo-de-prova (anel flutuante de consolidação); anéis de consolidação com áreas de 36,3 e 18,8 cm² para corpos-de-provas de áreas distintas; e placas porosas para drenagem da água.

O dispositivo de acondicionamento da amostra proposto para o equipamento é do tipo anelflutuante, isto é, espera-se que a fricção entre as paredes internas do anel e o solo seja menor do que no tipo de anel fixo. Nesse caso, para corposde-prova de solos de textura arenosa ou de textura argilosa de atividade alta, quando muito secos, se submetidos ao ensaio uniaxial, a fricção lateral não suporta o peso do anel, havendo o desprendimento do solo. 
(a)

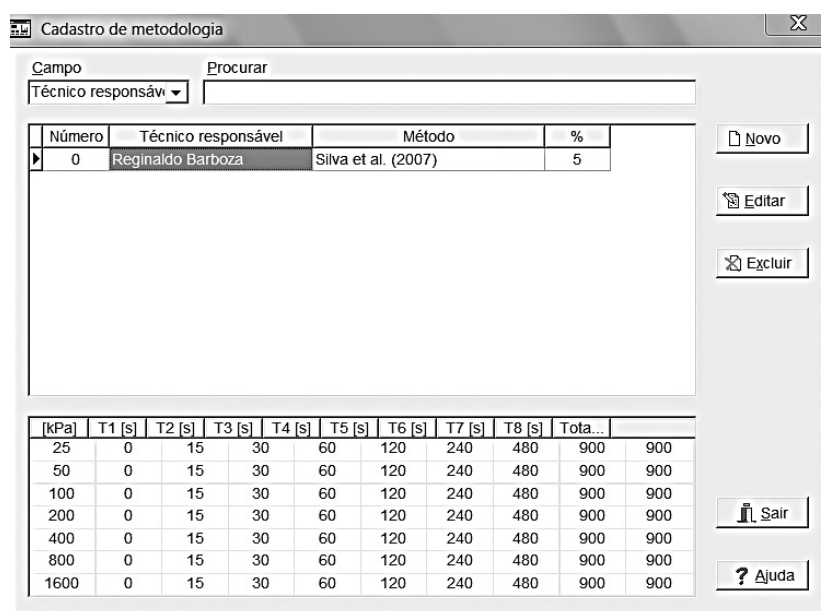

(b)

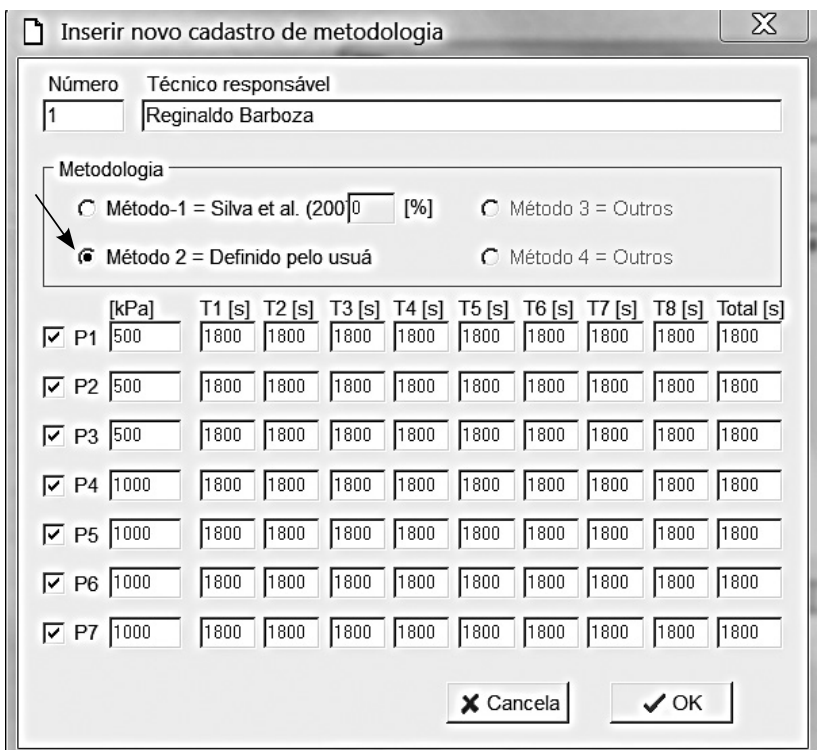

Figura 3. Tela exibindo opções para definir níveis de carregamento, índice relativo e tempo de consolidação. Na tela (a), detalhes para opção do ensaio de consolidação com sete carregamentos, conforme sugestão de Dias Júnior (1994), e valor do índice relativo (IR) de $5 \%$; na tela (b), uma segunda opção para definir carregamentos e tempos distintos, segundo necessidade do usuário.

De acordo com EM 1110-2-1906 (U.S. Army Engineer Manual, 1970), o dispositivo de anel flutuante só é satisfatório para solos coesos e duros, isto é, que permita o adequado acondicionamento do solo dentro do anel de consolidação. Em decorrência desses fatores e como já recomendado por Silva et al. (2007), além do anel de consolidação padrão (altura de $0,025 \mathrm{~m}$ e diâmetro de $0,063 \mathrm{~m}$ ), está sendo proposto um anel de consolidação de altura e diâmetro de
$0,05 \times 0,05 \mathrm{~m}$ para ensaios com solos mais soltos (arenosos) ou com predomínio de argilomineral 2:1, que, quando secos, apresentam elevado grau de contração (Figura 4). Entretanto, recomenda-se que para a sua utilização sejam conduzidos ensaios para comprovar se a nova relação entre o diâmetro e a altura não interfere no ensaio. Como vantagem do uso desse anel, as dimensões dele são as sugeridas como padrão pela Embrapa (2011), no uso em estudos e análises de rotinas de algumas propriedades físicas do solo, como a densidade do solo.

A calibração do equipamento, que foi feita ajustando-se força (F) em função da pressão aplicada na amostra $(\mathrm{Pa})$ permitiu obter a equação 1 $\left(F=0,0491^{* *}+0,3630^{* *} P a\right.$, em que $P a$ é a pressão na amostra; e $F$, a força), cujo coeficiente de correlação foi de $0,999^{* *}$. Essa alta precisão da relação entre esses dois parâmetros se deu por causa da sensibilidade da leitura da célula de carga, que é corrigida automaticamente por meio de rotinas programadas no CLP, cuja leitura em kgf apresenta uma precisão de $0,01 \%$ de fundo escala; isso significa que para a célula de carga de capacidade de $1.000 \mathrm{kgf}$ o erro máximo possível é de apenas 0,0001 kgf. Dessa forma, além da leitura da força ou pressão na amostra, que podem ser verificadas no display da IHM do CLP ou no monitor do computador, o usuário pode, ainda, fazer uso da equação 1 para definir novos níveis de força quando optar por corpo-de-prova de outras dimensões e, ou, outros níveis de pressão a serem aplicados na amostra.

\section{Princípio e funcionamento do equipamento}

O princípio e funcionamento do equipamento preconizam que um corpo-de-prova (amostra de solo indeformada) confinado lateralmente em anel de aço, com drenagem livre nas superfícies superior e inferior, seja submetido a incrementos sucessivos de pressão vertical. O funcionamento do equipamento se deve basicamente à atuação simultânea de dois conjuntos de dispositivos: "Conjunto pneumático", que tem a função de aplicar cargas instantâneas e manter o carregamento por tempos definidos sobre o corpo-de-prova; e "Conjunto de atuadores de força e deformação", que tem a função de informar e gerenciar a força, assim como o recalque do corpo-de-prova em milímetros.

O conjunto pneumático é alimentado por uma fonte de ar comprimido produzido por um compressor de baixa pressão e alto volume (pressão de trabalho de até 12 bares/175 psi), cujos níveis de pressão e tempos de compressão, previamente programados, são gerenciados por um software desenvolvido em linguagem de programação ladear, por meio do CLP com IHM incorporada.

Iniciado o ensaio, instantaneamente, a reguladora de pressão proporcional e a válvula direcional, atendendo a comandos programados em ladder no CLP e da IHM incorporada, permitem a 


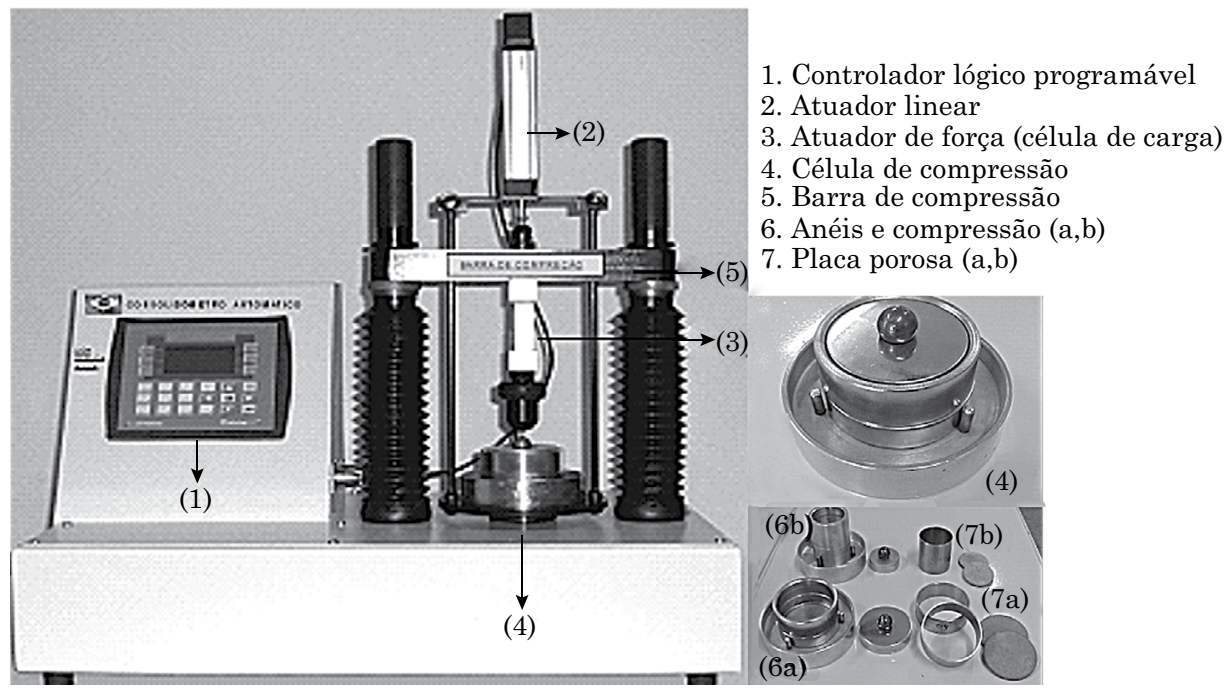

Figura 4. Consolidômetro automatizado com IHM incorporada e respectivos conjuntos de dispositivos e acessórios.

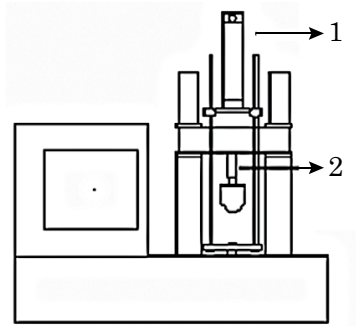

a) Vista frontal

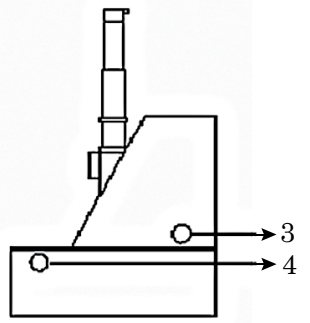

c) Vista lateral esquerda

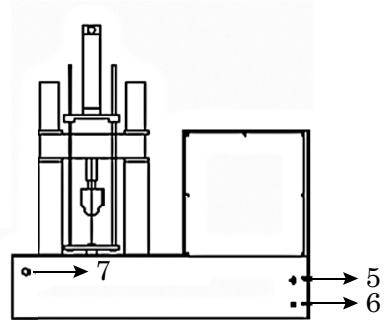

b) Vista traseira

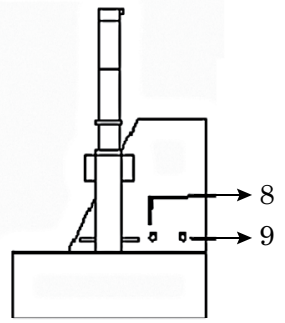

d) Vista lateral direita

1. Atuador linear;

2. Atuador de força (célula de carga);

3. Porta de comunicação;

4. Chave seletora de liga-desliga;

5. Fusível de $10 \mathrm{~A}$;

6. Alimentação 127 e 220 v;

7. Alimentação de ar comprimido;

8. Plugue de entrada do posicionador linear;

9. Plugue de entrada do atuado de força (célula de carga).

Figura 5. Cortes esquemáticos do equipamento e posicionamentos dos seus constituintes.

passagem do ar comprimido que corresponda aos respectivos níveis de pressão $(25,50$ 100, 200, 400, 800 e $1600 \mathrm{kPa})$ e força $(9,18,36,72,144.288$ e $572 \mathrm{kgf})$, que, nesse caso, por exemplo, trata-se de uma rotina-padrão programada e embarcada no

equipamento específico para o corpo-de-prova de $36 \mathrm{~cm}^{2}$, conforme sugestão de Dias Júnior (1994). O equipamento, contudo, é versátil e permite, também, a inclusão pelo usuário de outras rotinas, alterando parâmetros de força, pressão, tempo e dimensões do corpo-de-prova.

O ar comprimido, por sua vez, propulsiona o atuador pneumático de simples ação por membrana, que, por meio de um eixo, desloca um prato de estrutura metálica, onde está acondicionado o corpo-de-prova, contra uma estrutura rígida e fixa (barra de compressão). Assim, o corpo-de-prova é comprimido de maneira orientada e precisa e, portanto, a transmissão da pressão à estrutura do solo não é comprometida.

Entre a barra de compressão e o corpo-de-prova está posicionado o atuador de força (célula de carga), que monitora e corrige instantaneamente, por meio de conversores (módulos de expansão) incorporados ao CLP, os níveis de força correspondente a cada nível de pressão que está sendo aplicado no corpo-de-prova. Acima do corpo-de-prova e fixado à barra de compressão, o atuador de posição linear (régua digital de precisão de $0,0001 \mathrm{~mm}$ ) transmite, por meio de saídas analógicas ao CLP, os dados da deformação vertical do corpo-de-prova (recalque), que podem ser lidos em tempo real no display do controlador ou de um PC, ou ainda registrados para posterior processamento (Figuras 4, 5 e 6).

\section{Coleta e gerenciamento de dados}

O software desenvolvido (CA-Linker, v. 1.3) permite a comunicação entre o equipamento e o PC de forma instantânea (Figura 6), possibilitando a coleta, o armazenamento e o gerenciamento dos arquivos de dados de ensaios já realizados. O CA-Linker ao ser inicializado possibilita ao 


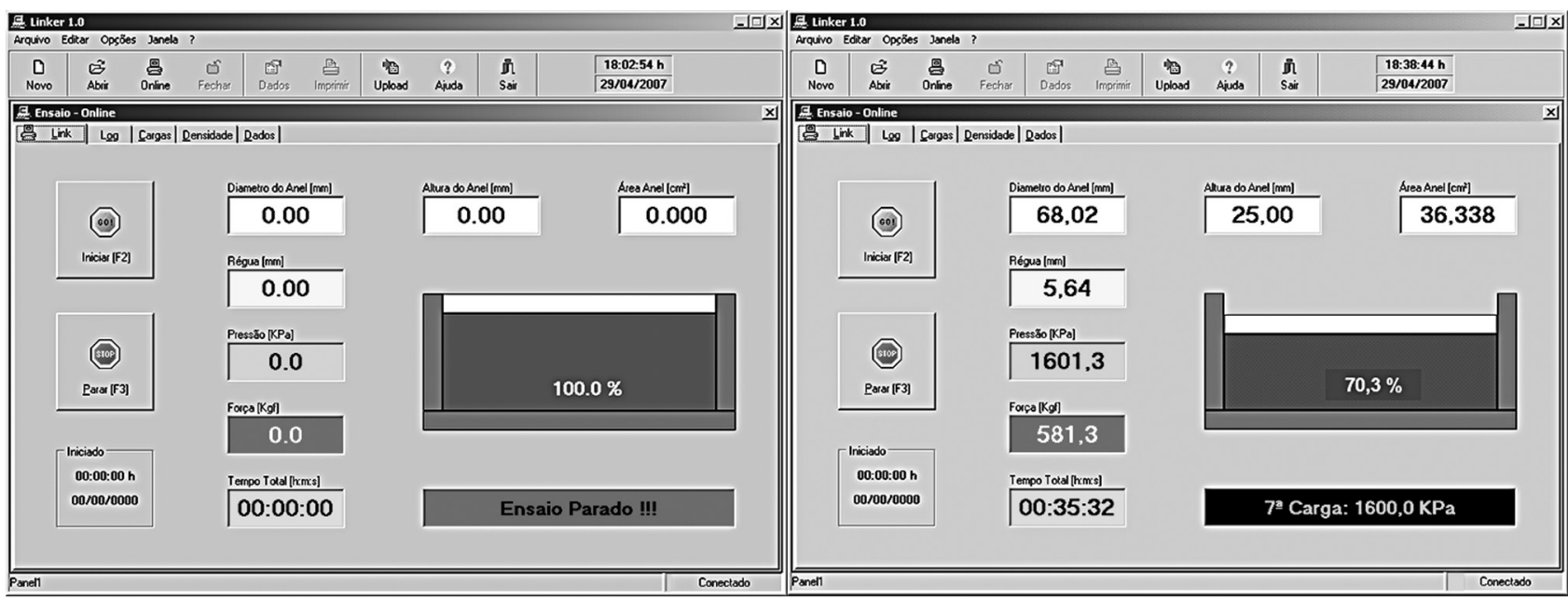

Figura 6. Informações do ensaio on-line do software CA-Linker permitindo a leitura de dados no início (à esquerda) e fim (à direita) de um ensaio realizado com o consolidômetro automatizado com IHM incorporada.

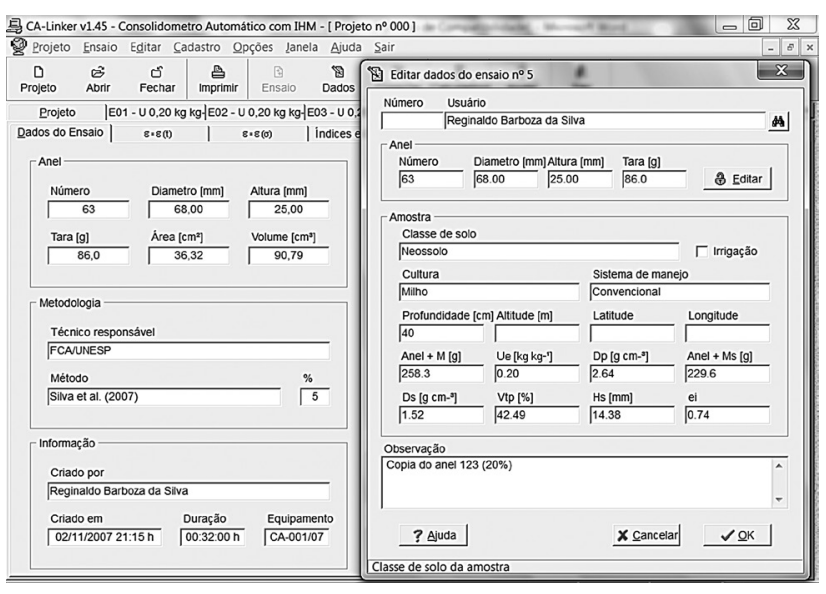

Figura 7. Detalhes da barra de ferramentas e janelas do software CA-Linker com recursos de edição de informações referentes ao "projeto" (à esquerda) e ao "ensaio" (à direita).

usuário, por meio de ícones de atalhos e de menus presentes na barra ferramentas, total interação com o equipamento, permitindo gerenciar projetos e ensaios, editar dados (antes e pós-ensaio), definir pressões e tempos de carregamentos, entre outros procedimentos (Figura 7). O CA-Linker permite, ainda, que todas as informações cadastrais, como dados referentes ao proprietário, propriedade, cultura, caracterização do solo, georreferenciamento da amostra, características do anel, metodologia utilizada, técnico responsável e, ou, usuário, sistema de manejo, entre outros, fiquem armazenadas em um banco de dados, possibilitando maior organização e rapidez na acessibilidade da informação.

O CA-Linker foi programado de tal forma para permitir maior rapidez, segurança, confiabilidade nos níveis de pressão aplicados ao corpo-de-prova e, principalmente, eliminar a interferência do técnico durante a realização do ensaio. $\mathrm{O}$ sistema gerencia os dispositivos (pneumáticos, eletrônico-digitais e atuadores de força e posição) incorporados ao equipamento, permitindo ao usuário o acompanhamento, em tempo real (status on-line), de todos os parâmetros envolvidos no ensaio de consolidação: deformação do corpo-de-prova $(\mathrm{mm})$, força $(\mathrm{kgf})$, pressão $(\mathrm{kPa})$, densidade do solo referente a cada pressão aplicada e tempo do ensaio, entre outras informações (Figura 6).

O CA-Linker processa os dados de força e deformação e, por meio de rotinas e pressuposições da mecânica se solo (divulgadas e disponibilizadas no meio acadêmico-científico), possibilita que sejam estimados os índices físicos e mecânicos, gerados pelo ensaio de consolidação. Significa dizer que o técnico, durante e imediatamente à finalização do ensaio, tem disponibilizado, em forma gráfica e de tabelas, valores de índice físicos e mecânicos decorrentes do ensaio de consolidação. De acordo com a figura 6 , é possível observar dois momentos: ensaio parado, aguardando definições de parâmetros; e ensaio sendo realizado

Quadro 2. Arquivo de saída de dados com valores de deformação em milímetros referente aos tempos de compressão para carga aplicada

\begin{tabular}{|c|c|c|c|c|c|c|c|c|}
\hline \multirow{2}{*}{ Pressão } & \multicolumn{8}{|c|}{ Tempo (s) } \\
\hline & $\mathbf{0}$ & 15 & 30 & 60 & 120 & 240 & 480 & 900 \\
\hline & \multicolumn{8}{|c|}{ Deformação (mm) } \\
\hline 25 & 0,00 & 0,27 & 0,30 & 0,32 & 0,35 & 0,35 & $\Gamma 0,37$ & - \\
\hline 50 & 0,37 & 0,60 & 0,62 & 0,62 & 0,65 & 0,67 & - & - \\
\hline 100 & 0,67 & 1,02 & 1,02 & 1,05 & 1,07 & $1,07 \leftrightarrows$ & - & - \\
\hline 200 & 1,07 & 1,42 & 1,45 & 1,47 & 1,50 & $1,52 \leftarrow$ & - & - \\
\hline 400 & 1,52 & 1,99 & 1,99 & 2,02 & 2,07 & $2,07 \leftrightarrow$ & - & - \\
\hline 800 & 2,07 & 2,57 & 2,64 & 2,64 & 2,64 & $2,67 \leftarrow$ & - & - \\
\hline 1.600 & 2,67 & 3,24 & 3,24 & 3,24 & 3,29 & 3,31 & - & - \\
\hline
\end{tabular}


em tempo real (on line) para a carga de $1.600 \mathrm{kPa}$. Verificam-se as leituras de pressão, força, deformação linear e volumétrica do corpo-de-prova, que podem ser acompanhadas instantaneamente, possibilitando um olhar mais crítico e detalhado durante a realização do ensaio.

\section{Avaliação do equipamento}

No quadro 2, está apresentado o resultado de um dos ensaios realizados com consolidômetro automatizado com IHM incorporada. Os valores de deformação em milímetros referente aos tempos de compressão para cada carregamento (nível de pressão) aplicado são exportados para um arquivo de saída no formato XLS. Observa-se que as deformações são registradas em razão da rotina incorporada ao software, que considerou o comparativo entre o valor que corresponde a $5 \%$ da deformação do tempo corrente e o valor referente à diferença de deformação do tempo corrente e o anterior (Quadro 1). Isso evidencia a atuação da IHM sobre o gerenciamento dos dispositivos pneumáticos, dos atuadores de força e da deformação.

Um ensaio que normalmente levaria $1 \mathrm{~h}$ e $45 \mathrm{~min}$, com a automação baixou para $56 \mathrm{~min}$. Contudo, considerando que o tempo mínimo por nível de pressão programado é de $4 \mathrm{~min}$, em amostras de um solo de textura média ou arenosa submetidas a baixos teores de água, e, portanto de baixa compressibilidade, o ensaio levaria no mínimo $28 \mathrm{~min}$.

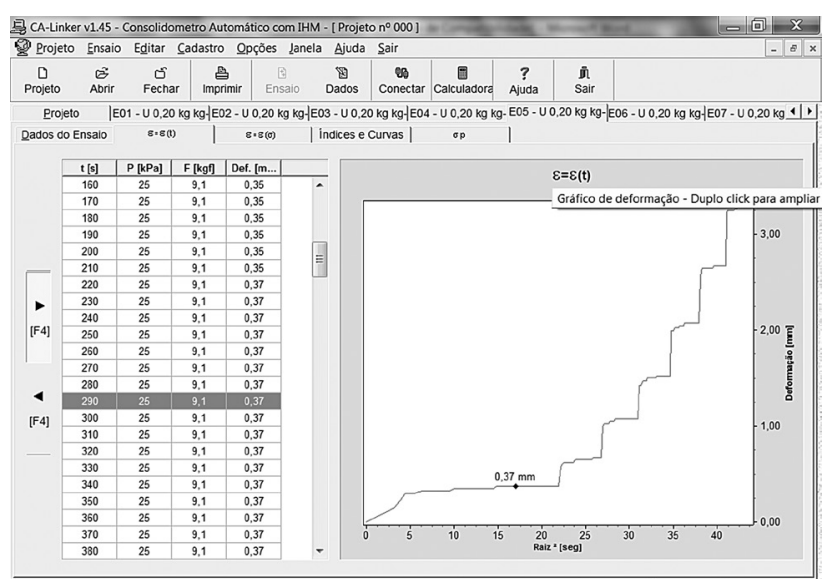

Figura 8. Interface gráfica apresentando em tempo real os valores de deformação em função do tempo para os diferentes níveis de carregamentos. As informações podem ser visualizadas na curva de deformação (direita) ou na tabela (esquerda) por causa do tempo para diferentes níveis de carregamentos. No detalhe, está destacado (no gráfico e na tabela) a deformação $(0,37 \mathrm{~mm})$ referente ao tempo de $290 \mathrm{~s}$ na pressão de $25 \mathrm{kPa}$ e força de $9,1 \mathrm{kgf}$ em um corpo-de-prova de área de $36,3 \mathrm{~cm}^{2}$.
Os valores de deformação em função do tempo são também acompanhados em tempo real por uma escala gráfica. Na figura 8, são apresentados o formato e o comportamento das curvas de deformação $(\mathrm{mm})$ por causa do tempo (s) a cada 10 s. Na "tela" (Figura 8), é identificado o ensaio com as curvas de deformação e a tabela de saída dos dados, em que são registradas as informações de tempo (em segundos), pressão (em $\mathrm{kPA}$ ), força (em kgf) e deformação (em mm). Esse é outro recurso incorporado ao equipamento, que permite graficamente acompanhar todo o processo

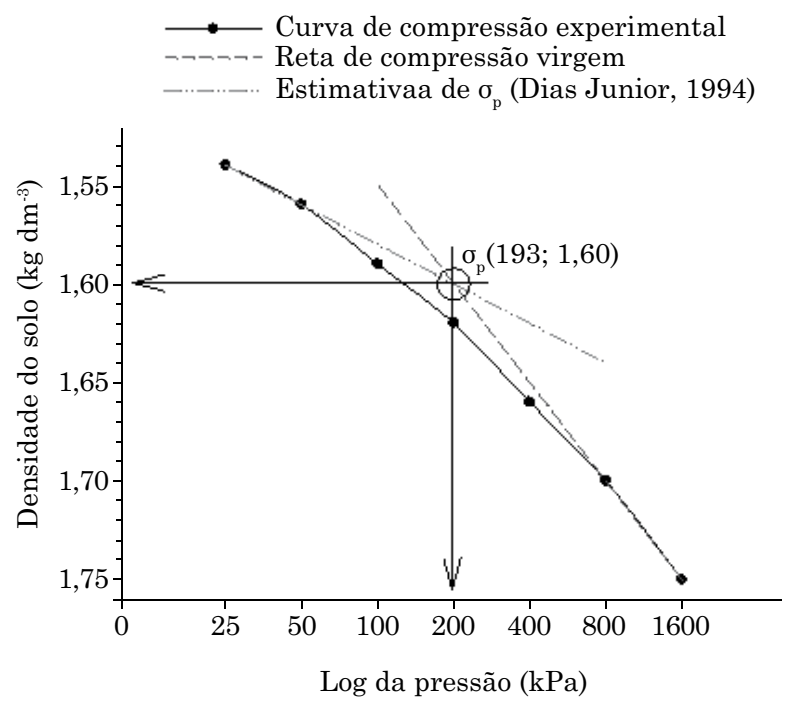

Figura 9. Curva de compressão experimental e estimativa da pré-consolidação e da densidade do solo, conforme sugestão da literatura (Dias Júnior e Pierce, 1996).

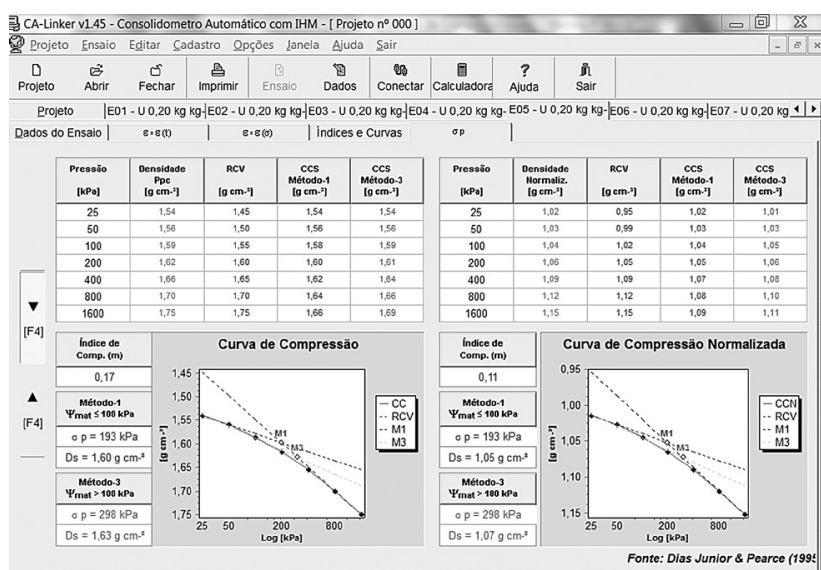

Figura 10. Interface gráfica apresentando em tempo real o resultado final do ensaio uniaxial (curva de compressão do solo com dados naturais e normalizados) e os principais índices físicos e mecânicos do solo: pressão de pré-consolidação (op), densidade do solo (Ds) e índice de compressão (m). 
de deformação do corpo-de-prova e observar e confirmar se o tempo de consolidação está atendendo à sugestão de Taylor (1948).

Os dados de deformação para cada tempo, obtidos segundo Taylor (1948), depois de convertidos para densidade $\left(\mathrm{kg} \mathrm{dm}^{-3}\right)$, são plotados em função das suas respectivas pressões (carregamentos), permitindo plotar em tempo real a curva de compressão do solo e finalizá-la concomitante com ensaio (Figura 9). É possível notar o comportamento harmonioso da curva de compressão experimental e a estimativa da pressão de pré-consolidação e sua respectiva densidade do solo. $\mathrm{O}$ procedimento utilizado foi o método 1 (M1) de Dias Júnior e Pierce (1996), que, por causa da sua grande aceitação e utilização na maioria dos estudos realizados no Brasil, além do método 3 (M3) dos respectivos autores, foi embarcado nas rotinas do equipamento como sugestão para estimativa de pré-consolidação.

Segundo Larson et al. (1980) e Larson e Gupta (1980), a curva de compressão tem sido utilizada para simular tais reduções e relacionar o logaritmo da pressão aplicada; e alguma propriedade do solo dependente do arranjo das partículas e, ou, agregados do solo, como o índice de vazios ou a densidade do solo (Casagrande, 1936; Holtz e Kovacs, 1981).

$\mathrm{Na}$ figura 10 ("tela"), é possível verificar o resultado final do ensaio uniaxial. Nas tabelas, são informados os dados estimados de densidade do solo $\left(\mathrm{kg} \mathrm{dm}^{-3}\right)$, que em função dos níveis de pressão $(\mathrm{kPa})$ permitem o ajuste das retas de compressão virgem (RCV) e das curvas de compressão secundária (CCS), aqui obtidas pelos métodos M1 e M3, conforme sugestão de Dias Júnior e Pierce (1996). É observado que duas curvas de compressão são geradas, sendo uma delas com valores de densidades do solo normalizadas. Sugestão essa para que a pressão de pré-consolidação não seja influenciada

Quadro 3. Alguns dos índices volumétricos de um corpo-de-prova submetido a $0,20 \mathrm{~kg} \mathrm{~kg}^{-1}$, gerados durante o ensaio de consolidação com o consolidômetro automatizado

\begin{tabular}{rcccccc}
\hline \multicolumn{1}{c}{ Pr } & Def & ÍV & Alt & Vol & Pt & Red Por \\
\hline $\mathbf{k P a}$ & $\mathbf{c m}$ & & $\mathbf{c m}$ & $\mathbf{d m}^{3}$ & & \multicolumn{2}{c}{$\%$} \\
\cline { 5 - 7 } 25 & 0,37 & 0,71 & 24,63 & 89,45 & 41,63 & 2,03 \\
50 & 0,67 & 0,69 & 24,33 & 88,36 & 40,91 & 3,73 \\
100 & 1,07 & 0,66 & 23,93 & 86,91 & 39,92 & 6,05 \\
200 & 1,52 & 0,63 & 23,48 & 85,27 & 38,77 & 8,76 \\
400 & 2,07 & 0,59 & 22,93 & 83,27 & 37,30 & 12,22 \\
800 & 2,67 & 0,55 & 22,33 & 81,10 & 35,62 & 16,18 \\
1.600 & 3,31 & 0,51 & 21,69 & 78,77 & 33,72 & 20,65 \\
\hline
\end{tabular}

Pr: pressão aplicada; Def: deformação; IV: índice de vazios; Al: altura; Vol: volume; Pt: porosidade total; Red Por: redução da porosidade do corpo-de-prova. pela densidade do solo inicial. Para cada curva são estimados, portanto, de acordo com cada método (M1 e M3), os valores de pré-consolidação e sua respectiva densidade do solo, além do índice de compressão $(\mathrm{m})$, que reflete a suscetibilidade do solo à compactação.

$\mathrm{O}$ equipamento possibilita, ainda, por meio de suas rotinas, um resumo (Quadro 3) dos principais índices físicos do corpo-de-prova, gerados após o ensaio uniaxial. Informações de deformação $(\mathrm{cm})$, índice de vazios, altura $(\mathrm{cm})$, volume do corpo-de-prova $\left(\mathrm{cm}^{3}\right)$ e volume total de poros (\%) para cada nível de pressão aplicado $(\mathrm{kPA})$ possibilitam ao usuário maior interpretação da relação de massa e volume da sua amostra e, por conseguinte, melhores inferências a despeito dos propósitos do seu estudo.

De acordo com o quadro 3, verificam-se aumento dos valores de deformação e redução dos valores de índice de vazios, altura, volume e porosidade, assim como a sua redução em razão dos níveis crescentes de pressão aplicados ao corpo-de-prova. Tal comportamento evidencia o bom desempenho do conjunto pneumático e dos atuadores de força e deformação e a sua interação com as rotinas e comandos programados em ladder e gerenciados pelo CLP e IHM pelo CA-Linker.

\section{CONCLUSÕES}

A aplicação do CLP com IHM incorporada permitiu o desenvolvimento e automação do consolidômetro. O desempenho e a eficiência do conjunto de dispositivos (pneumáticos, eletrônico-digital e atuadores de força e deformação) foram comprovados pelos excelentes resultados dos valores de deformação em razão do tempo e comportamento das curvas de compressão, geradas dos ensaios uniaxiais.

Os softwares e as rotinas do equipamento apresentam uma interface amigável e possibilitam, instantaneamente, o monitoramento, a coleta e processamento de dados do Consolidômetro Automático com IHM. Associado aos recursos do controlador lógico programável (CLP), o software $C A$-Linker permite ao usuário controle total sobre os dispositivos pneumáticos e eletrônico-digital e dos atuadores de força e posição.

O Consolidômetro Automático com IHM dispensa a dedicação exclusiva de um técnico no desempenho de rotinas de registro e processamento de dados, necessários para estimativa dos principais índices físicos e mecânicos do solo, assim como a sistematização de gráficos e tabelas. 


\section{REFERÊNCIAS}

Ajayi AE, Dias Júnior MS, Curi N, Okunola A, Souza TT, Pires BS. Assessment of vulnerability of Oxisols to compaction in the Cerrado region Brazil. Pedosphere. 2010;20:252-60.

Ajayi AE, Dias Júnior MS, Curi N, Olufayo AA. Soil colour as a simple indicator of load bearing capacity in Brazilian Latosols. J Indian Soc Soil Sci. 2011;59:14-21.

Ajayi AE, Dias Júnior MS, Curi N, Oladipo I. Compressive response of some agricultural soils influenced by the mineralogy and moisture. Int. Agrophys. 2013;27:239-46.

Araujo-Junior CF, Dias Junior MS, Guimarães PTG. Resistência à compactação de um Latossolo cultivado com cafeeiro, sob diferentes sistemas de manejo de plantas invasoras. R Bras Ci Solo. 2008;32:23-32.

Araujo Júnior CF, Dias Júnior MS, Guimarães PTC, Alcântara EN. Capacidade de suporte de carga e umidade crítica de um Latossolo induzida por diferentes manejos. R Bras Ci Solo. 2011;35:115-31.

Bowles JE. Engieneering properties of soils and their measurements. $3^{\text {a }}$.ed. Auckland: McGraw-Hill; 1986. 218p.

Casagrande, A. The determination of pre-consolidation load and its practical significance. In: Proceedings of the $1^{\text {st }}$ International Conference on Soil Mechanics and Foundation Engineering, 1936; Cambridge. Cambridge; 1936. v.3, p.60-4.

Dias Júnior MS, Pierce FJ. O processo de compactação do solo e sua modelagem. R Bras Ci Solo. 1996;20:175-82.

Dias Júnior MS, Pierce FJ. A simple procedure for estimating preconsolidation pressure from soil compression curves. Soil Technol. 1995;8:139-51.

Dias Júnior MS. Compression of three soils under long- term tillage and wheel traffic, ast [thesis]. Lansing: Michigan State University; 1994

Dias Júnior MS, Leite FP, Lasmar Júnior E, Araujo-Júnior CF. Traffic effects on the soil preconsolidation pressure due to eucalyptus harvest operations. Sci Agric. 2005;62:248-55.

Empresa Brasileira de Pesquisa Agropecuária - Embrapa. Manual de métodos de análise de solo. Rio de Janeiro; 2011. p.43-56.

Empresa Brasileira de Pesquisa Agropecuária - Embrapa. Sistema brasileiro de classificação de solos. Rio de Janeiro: Centro Nacional de Pesquisa de Solos; 2013.

Figueiredo GC, Silva AP, Tormena CA, Giarola NFB, Moraes SO, Almeida BG. Desenvolvimento de um consolidômetro pneumático: Modelagem da compactação, penetrometria e resistência tênsil de agregados de solo. R Bras Ci Solo. 2011;35:389-402.

Holtzs RD, Kovacs WD. An introduction to geotechnical engineering. Englewood Cliffs: Prentice-Hall; 1981.

Iori P, Silva RBS, Dias Júnior MS, Lima JM. Pressão de preconsolidação como ferramenta de análise da sustentabilidade estrutural de classes de solos com diferentes usos. R Bras Ci Solo. 2012a;36:1448-56.

Iori $\mathrm{P}$, Dias Júnior MS, Silva RB. Resistência do solo à penetração e ao cisalhamento em diversos usos do solo em áreas de preservação permanente. Biosci J. 2012b;28:185-95.

Iori P, Dias Junior MS, Ajayi AE, Guimarães PTG, Pais PSM, Andrade MLC. Comparison of field and laboratory models of the load bearing capacity in coffee plantations. Ci Agrotec. 2013;37:130-7.

Kamimura KM, Dias Júnior MS, Guimarães PTG, Santos GR, Oliveira MS. Load bearing capacity of a Red-Yellow Latosol in a coffee plantation. R Bras Ci Solo. 2012;36:1457-65.

Kamimura KM, Santos GR, Oliveira MS, Dias Junior MS, Guimarães PTG. Spatial variability of the physical properties of a Red Yellow Latosol under coffee. R Bras Ci Solo. 2013;37:877-88.

Larson WE, Gupta SC. Estimating critical stress in unsaturated soils from changes in pore water pressure during confined compression. Soil Sci Soc Am J. 1980;44:1127-32.

Larson WE, Gupta SC, Useche RA. Compression of agricultural soil from eight soil orders. Soil Sci Soc Am J. 1980;44:450-7.

Martins PCC, Dias Júnior MS, Carvalho JS, Silva AR, Fonseca SM. Levels of induced pressure and compaction as caused by forest harvesting operations. Cerne. 2013;19:83-91.

Pais PSM, Dias Júnior MS, Santos GA, Dias AC, Guimarães PTG, Alcântara EN. Compactação causada pelo manejo de plantas invasoras em Latossolo Vermelho-Amarelo cultivado com cafeeiros. R Bras Ci Solo. 2011;35:1949-57.

Pais, PSM, Dias Júnior MS, Dias AC, Iori P, Guimarães PTG, Santos GA. Load-bearing capacity of a Red-Yellow Latosol cultivated with coffee plants subjected to different weed managements. Ci. Agrotec. 2013;37:145-51.

Peng XH, Horn R, Zhang B, Zhao QG. Mechanisms of soil vulnerability to compaction of homogenized and recompacted Ultisols. Soil Till Res. 2004;76:125-37.

Pires BS, Dias Júnior MS, Rocha WW, Araújo Júnior CF, Carvalho RCR. Modelos de capacidade de suporte de carga de um Latossolo Vermelho-Amarelo sob diferentes usos e manejos. R Bras Ci Solo. 2012;36:635-42.

Severiano EC, Oliveira GC, Dias Júnior MS, Costa KAP, Silva FG, Ferreira Filho SM. Structural changes in Latosols of the cerrado region: II - soil compressive behavior and modeling of additional compaction. R Bras Ci Solo. 2011;35:783-91.

Severiano EC, Oliveira GC, Dias Júnior MS, Curi N, Costa KAP, Carducci CE. Preconsolidation pressure, soil water retention characteristics, and texture of Latosols in the Brazilian Cerrado. Soil Res. 2013;51:193-202.

Severiano, EC, Oliveira GC, Dias Júnior MS, Oliveira LFC, Castro MB. Pressão de preconsolidação e intervalo hídrico ótimo como indicadores de alterações estruturais do solo em decorrência das operações de colheita da cana-de-açúcar. R Bras Ci Solo. 2008;32:1419-27.

Silva RB, Dias Junior MS, Santos FL, Franz CAB. Influência do preparo inicial sobre a estrutura do solo quando da adoção do sistema plantio direto, avaliada por meio da pressão de preconsolidação. R Bras Ci Solo. 2003a;23:219-26.

Silva RB, Lanças KP, Masquetto BJ. Consolidômetro: Equipamento pneumático-eletrônico par avaliação do estado de consolidação do solo. R Bras Ci Solo. 2007;31:617-5.

Silva RB, Masquetto BJ. CA-Linker: Sistema para monitoramento, aquisição e processamento de parâmetros físicos e mecânicos em tempo real, gerados em ensaios de consolidação uniaxial. In: Anais do $32^{\circ}$ Congresso Brasileiro de Ciência do Solo [CD-ROM]; 2009; Fortaleza. Fortaleza: Universidade Federal do Ceará; 2009. 
Silva RB, Iori P, Silva FAM, Dias Júnior MS. Modelagem e determinação do estado crítico de consolidação a partir da relação massa e volume em solos canavieiros. R Ci Agron. 2010;33:376-89.

Silva RB, Dias Junior MS, Silva FAM, Fole SM. O tráfego de máquinas agrícolas e as propriedades físicas, hídricas e mecânicas de um Latossolo dos cerrados. R Bras Ci Solo. 2003b;27:973-83.

Silva RB, Lanças KP, Miranda EEV, Silva FAM, Baio FHR. Estimation and evaluation of dynamic properties as indicators of changes on soil structure in sugarcane fields of Sao Paulo State Brazil. Soil Till Res. 2009;103:265-70.

Souza GS, Souza ZM, Silva RB, Araújo FS, Barbosa RS. Compressibilidade do solo e sistema radicular da cana-de-açúcar em manejo com e sem controle de tráfego. Pesq Agropec Bras. 2012;47:603-12.

Taylor DW. Fundamentals of soil mechanics. New York: John Wiley \& Sons; 1948.

United States Army Engineer Manual. EM 1110-2-1906. Laboratory Soils Testing. Washington; 1970. 